\title{
A Novel Stochastic Framework for the Optimal Placement and Sizing of Distribution Static Compensator
}

\author{
Reza Khorram-Nia*, Aliasghar Baziar, Abdollah Kavousi-Fard \\ Department of Electrical and Computer Engineering, Safashahr Branch, Islamic Azad University, Safashahr, Iran. \\ Email: ${ }^{*}$ r.khorramnia@gmail.com, bazyar.aliasghar@gmail.com, abdollah.kavousifard@gmail.com \\ Received February $28^{\text {th }}, 2013$; revised April 29 ${ }^{\text {th }}, 2013$; accepted May $6^{\text {th }}, 2013$ \\ Copyright (C) 2013 Reza Khorram-Nia et al. This is an open access article distributed under the Creative Commons Attribution Li- \\ cense, which permits unrestricted use, distribution, and reproduction in any medium, provided the original work is properly cited.
}

\begin{abstract}
This paper proposes a new stochastic framework based on the probabilistic load flow to consider the uncertainty effects in the Distribution Static Compensator (DSTATCOM) allocation and sizing problem. The proposed method is based on the point estimate method (PEM) to capture the uncertainty associated with the forecast error of the loads. In order to explore the search space globally, a new optimization algorithm based on bat algorithm (BA) is proposed too. The objective functions to be investigated are minimization of the total active power losses and reducing the voltage deviation of the buses. Also to reach a proper balance between the optimization of both the objective functions, the idea of interactive fuzzy satisfying method is employed in the multi-objective formulation. The feasibility and satisfying performance of the proposed method is examined on the 69-bus IEEE distribution system.
\end{abstract}

Keywords: DSTATCOM Allocation; Uncertainty; Multi-Objective Optimization; 2 m Point Estimate Method

\section{Introduction}

According to the failure statistics, the distribution systems play the most significant role in the unavailability of electrical services to the consumers [1,2]. Therefore, any planning or technique which can improve the total condition of the distribution systems should be assessed precisely such that the maximum efficiency and profit is achieved. In this regard, the utilities have been looking for new methods to improve the total system electrical services from both operation and planning points of view. Among the most well-known and popular methods can be named the optimal management of the shunt capacitors, shunt reactors, automatic voltage regulator, series capacitors or recently distribution network flexible AC transmission system (DFACTS) technologies such as distribution static compensator (DSTATCOM) [3].

In comparison to the other reactive power compensation devices, DSTATCOM has especial useful characteristics such as low harmonic production, low power losses, high regulatory ability and small size [4]. In addition, in comparison to some popular reactive power compensations methods such as shunt capacitor placement strategy or series capacitors, the DSTATCOM does not have any operational issues such as resonance or transient har-

"Corresponding author. monics as which are caused by switching. Another useful and significant aspect of the DSTATCOM is that it can clean up the voltage from any unbalance or harmonic distortion [5]. In fact, the DSTATCOM can compensate the load demand locally such that in the case of load variations it is regulated automatically. In the long time, the total load consumption in the power system is increased. Here again the DSTATCOM can be the most effective device to maximize the power system loadability, stability and reactive power compensation when reducing the resistive losses. In the area of the area of power quality, it will improve the quality of the electrical services by improving the flicker suppression, voltage regulation and voltage balancing [6].

According to the above discussion, it is deduced easily that the DSTATCOM device can play a significant role in the optimal operation and management of the future distribution networks. From the utility point of view, the most significant issue is to reduce the amount of MW power losses by reducing the resistive losses [7-10]. Another significant target is to improve the network power quality by improving the voltage of the buses. Improving the bus voltage can improve the electrical services by reducing the costs of damaging sensitive electrical devices as well as reducing the number of interruptions in the system. The significance of this objective function 
can be seen in several literatures [11-13]. Nevertheless, in the area of DSTATCOM allocation there is yet very little works available to see its effect on the distribution network from different aspects. In [14], the DSTATCOM allocation problem is assessed considering the objective functions of active power losses and voltage profile simultaneously. Nevertheless, neglecting the uncertainty associated with the active and reactive loads is a big shortage of the analysis which can reduce the dependability of the final results. In [15], the optimal DSTAT$\mathrm{COM}$ allocation and sizing is investigated to mitigate the voltage fluctuations in the network. Here, the simultaneous effect of the distribution generation (DG) on the optimal DSTATCOM allocation is assessed. However, again the uncertainty of the random variables is neglected here.

Therefore, the main purpose of this paper is to propose a new stochastic framework based on $2 \mathrm{~m}$ point estimate method (PEM) to capture the uncertainty associated with the active and reactive loads in the analysis. The proposed stochastic method will convert the stochastic DSTATCOM allocation problem to $2 \mathrm{~m}$ equivalent deterministic problems with different probabilities. In order to model the uncertainty of the loads, the normal distribution function with zero mean value is considered for each load value. The $2 \mathrm{~m}$ PEM will replace each random variable (here active and reactive loads) with two new concentrations points using just the first few statistical moments of the random variable. Therefore, for a problem with $m$ number of random variables, $2 \mathrm{~m}$ runs of the deterministic load flow are required to solve the probabilistic problem. The main popularity of $2 \mathrm{~m}$ PEM roots in the very little data which are required about the random variables of the problem. The objective functions which are investigated in the analysis are the total active power losses and the voltage profile. In order to handle both of the objective functions, a novel interactive fuzzy satisfying method is proposed. Since the investigated problem considering the uncertainty of the active and reactive loads is a complex and nonlinear optimization problem, it requires a powerful tool to escape from local optima as well as premature convergence. Therefore, a new optimization method based on modified bat algorithm (MBA) is proposed to explore the search space globally. In order to see the effectiveness and satisfying performance of the proposed method, the 69-bus IEEE radial distribution system is chosen as the case study. The rest of the paper is organized as follows: in Section 2, the objective functions and the relevant constraints are explained. In Section 3, the DSTATCOM modeling is described. In Section 4, the $2 \mathrm{~m}$ PEM is described completely. In Section 5, the optimization technique and the multi-objective formulation are explained. The simulation results are shown in Section 6. Finally, in Section 7, the main remarks and conclusions are discussed.

\section{Problem Formulation}

In this section, the objective functions and the relevant equality and inequality constraints are described completely.

\subsection{Objective Functions}

Minimization of the Active Power Losses $\left(f_{1}\right)$ : The active power losses objective function is the summation of the resistive power losses of the network branches. Mathematically, total active power losses can calculated as follows:

$$
f_{1}(X)=P_{\text {loss }}(X)=\sum_{i=1}^{N_{b r}} R_{i} \times\left|I_{i}\right|^{2}
$$

where $R_{i}$ is the resistance of $i^{\text {th }}$ branch, $I_{i}$ is the current of $i^{\text {th }}$ branch, $N_{b r}$ is the number of branches and $X$ is the control vector.

Minimization of the Voltage Deviation $\left(f_{2}\right)$ : The voltage profile of the system is improved by reducing the maximum voltage deviation of the buses from the nominal voltage value. Therefore, this target can be evaluated as follows:

$$
f_{2}(X)=d_{\text {volt }}(X)=\max \left\{\left|1-V_{\text {min }}\right|,\left|1-V_{\text {max }}\right|\right\}
$$

where $V_{\min }$ and $V_{\max }$ are the minimum and the maximum value of voltage magnitudes of $i^{t h}$ bus.

\subsection{Limits and Constraints}

Maximum Power Flow Constraint: This limit is associated with the maximum power transfer capacity of the distribution lines which should be observed during the optimization process.

$$
\left|P_{i j}^{\mathrm{Line}}\right|<P_{i j \text {,max }}^{\mathrm{Line}}
$$

where $P_{i j}^{\text {Line }}$ is the active power flow over the distribution lines of buses $i$ and $j$. Also, $P_{i j \text { max }}^{\text {Line }}$ is the maximum active power flow which is allowed to flow between the buses $i$ and $j$.

Distribution Power Flow Equations: This limitation consists of two power flow equations which can be considered as an equality constraint.

$$
\begin{gathered}
P_{i}=\sum_{i=1}^{N_{\text {bus }}} V_{i} V_{j} Y_{i j} \cos \left(\theta_{i j}-\delta_{i}+\delta_{j}\right) \\
Q_{i}=\sum_{i=1}^{N_{\text {bus }}} V_{i} V_{j} Y_{i j} \sin \left(\theta_{i j}-\delta_{i}+\delta_{j}\right)
\end{gathered}
$$

where $V_{i}$ is the voltage magnitude of $i^{\text {th }}$ bus, $\delta_{i}$ is the voltage angle of $i^{t h}$ bus, $Y_{i j}$ is the admittance magnitude between the buses $i$ and $j, \theta_{i j}$ is the admittance angle between the buses $i$ and $j, N_{\text {bus }}$ is the number of buses, $P_{i}$ is the net active power injection to the $i^{t h}$ bus and $Q_{i}$ 
is the net reactive power injection to the $i^{\text {th }}$ bus.

Bus Voltage Constraints: During the optimization process, the voltage level of the buses should be preserved in the pre-determined limited values as follows:

$$
V_{\min } \leq V_{i} \leq V_{\max }
$$

Feeder Current Limitation: The main feeders of the network can supply a maximum current magnitude as follows:

$$
\left|I_{f, i}\right| \leq I_{f, i}^{\max } ; i=1,2, \cdots, N_{f}
$$

where $I_{f, i}$ is the current of the $i^{\text {th }}$ feeder, $I_{f, i}^{\max }$ is the maximum current of the $i^{\text {th }}$ feeder and $N_{f}$ is the number of feeders.

\section{DSTATCOM Modeling}

The Static Synchronous Compensator (STATCOM) as a member of the Flexible AC Transmission Systems (FACTS) devices is a regulating power utility which is connected to the power system in shunt mode. Once the STATCOM is used in the voltage level of distribution system is called Distribution STATCOM or shortly DSTATCOM. The DSTATCOM device works based on the power electronics voltage source converters and can either produce or consume the reactive power of the electrical network at the coupling point. Nevertheless, in the case of using a power source, it can also provide active power simultaneously. In the DSTATCOM, the voltage level is generated from a DC capacitor. Therefore, the direction and amount of reactive power is determined by the magnitude of the voltage source. In the case that the magnitude of the voltage source is higher than that of the connection point, the DSTATCOM will produce reactive power and so will work as a variable capacitor. However, if the magnitude of the voltage source is lower than the voltage of the connection point, the DSTAT$\mathrm{COM}$ will work as a reactor and so will absorb reactive power.

In the steady state load flow analysis, the accurate DSTATCOM model should consist of the steady state power losses including the transformer and inverter power losses. Technically, the load flow model of the STATCOM is considered to be appropriate for the DSTATCOM device [14]. Therefore, considering the bus $i$ with the active and reactive load values of $P_{L i}+j Q_{L i}$, the DSTATCOM can be supposed as a new PV bus (we name it bus $j$ ) with fixed voltage value which is connected to the bus $i$. Since we have supposed that there is no power source connected to the DSTATCOM, thus it can just supply reactive power in the network. In the other words, the amount of active power production is assumed zero. In order to model the active power losses of the transformer connection and the inverter, the series reactance and resistance $R_{T}+j X_{T}$ are utilized [4]. The schematic diagram of the model is shown in Figure 1.

\section{Uncertainty Modeling}

In this section, a stochastic framework based on $2 \mathrm{~m}$ PEM is proposed to consider the uncertainty associated with the forecast error of the active and reactive loads. In comparison to the other well-known methods in the area, the $2 \mathrm{~m}$ PEM requires little statistical data of the probability density function of the random variables. Mathematically, the load flow equations can be described as follows [15]:

$$
S=F(z)
$$

where $z$ is the input uncertain vector and $S$ is the output uncertain vector. The main idea behind the $2 \mathrm{~m}$ PEM is to replace the random variable $z_{l}$ (with probability of $f_{z l}$ ) with two concentration points $z_{l, 1} \& z_{l, 2}$ as follows:

$$
z_{l, k}=\mu_{z_{l}}+\xi_{l, k} \cdot \sigma_{z_{l}} ; k=1,2
$$

where $\mu_{z_{l}}$ is the mean value of the random variable $z_{l}$ and $\sigma_{z_{l}}$ is its standard deviation. Here $\zeta_{l, k}$ is called the standard location and is evaluated is as follows:

$$
\xi_{l, k}=\frac{\lambda_{l, 3}}{2}+(-1)^{3-k} \sqrt{m-\left(\lambda_{l, 3}^{2} / 2\right)^{2}}, k=1,2
$$

The impact of the two locations $z_{l, 1} \& z_{l, 2}$ on the output variable is determined by the two weighting factors $\omega_{l, 1}$, $\omega_{l, 2}$ respectively. This process is shown in Figure 2.

According to Figure 2, the location moments $z_{l, 1} \&$ $z_{l, 2}$ are transferred to the output data $S_{l, 1} \& S_{l, 2}$ via Equation (7). Finally, the skewness coefficient $\left(\lambda_{l, 3}\right)$ as the third central moment is calculated as bellow:

$$
\lambda_{l, 3}=\frac{E\left[\left(z_{l}-\mu_{z_{l}}\right)^{3}\right]}{\left(\sigma_{z_{l}}\right)^{3}}
$$

Here $E$ is the expected value. In order evaluate the standard deviation of $S_{i}$, the bellow equation should be evaluated:

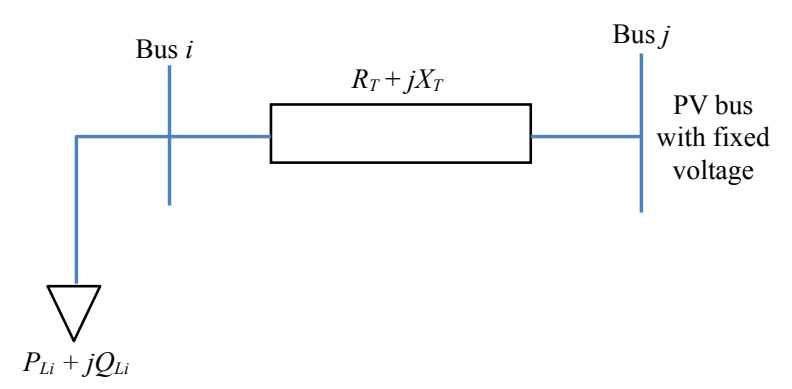

Figure 1. Schematic diagram of DSTATCOM model connected to bus $i$. 


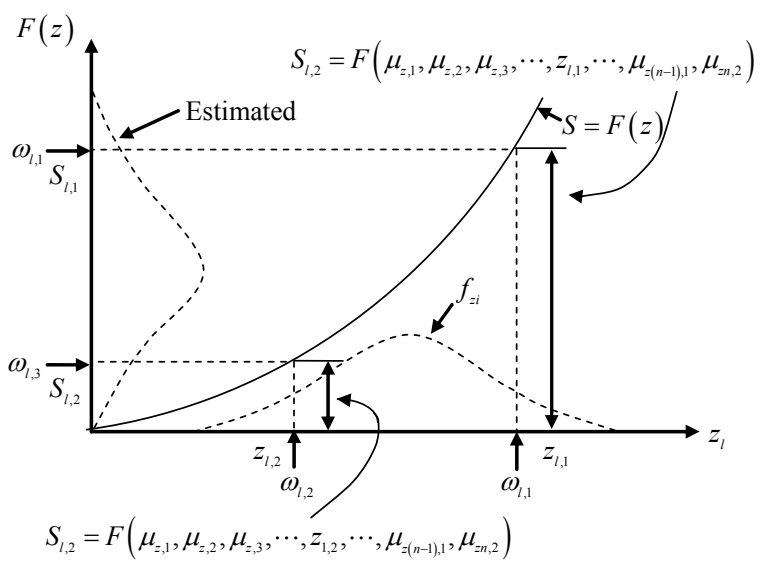

Figure 2. Schematic representation of 2 m PEM.

$$
\sigma=\sqrt{\operatorname{var}\left(S_{i}\right)}=\sqrt{E\left(S_{i}^{2}\right)-\left[E\left(S_{i}\right)\right]^{2}}
$$

\section{Optimization Technique}

In this section, the proposed modified BA as well as the interactive fuzzy satisfying method based on membership function is described.

\subsection{Original Bat Algorithm}

The BA is a population based optimization algorithm which was first introduced by Xin-She Yang in 2010 [16]. The BA simulates the searching ability of the bat animals for the food by using the echolocation phenomena. In definition, the echolocation is defined as the process of sending a signal to the environment and after that waiting to hear its echo. The BA is based on 3 main ideas [16]: 1) the distance is sensed by the echolocation phenomena; 2) each bat in the search space $\left(X_{i}\right)$ flying with the velocity of $v_{i}$ will produce a signal with frequency fre $e_{i}$, the wavelength $\Gamma_{i}$ and loudness $A_{i}$; and 3 ) it is supposed that the loudness $A_{i}$ can vary from the initial large value $A_{0}$ to its specific minimum value $A_{\min }$. Initially, the $\mathrm{BA}$ population is generated randomly. After evaluating the objective function for each bat, the entire population is updated in each iteration $k$ as follows:

$$
\begin{aligned}
& \text { fre }_{i}=\text { fre }_{\text {min }}+\left(\text { fre }_{\text {max }}-\text { fre }_{\text {min }}\right) \rho \\
& v_{i}^{k+1}=v_{i}^{k}+\left(X_{i}^{k}-X_{\text {gbest }}\right) \text { fre }_{i} \\
& X_{i}^{k+1}=X_{i}^{k}+v_{i}^{k+1}
\end{aligned}
$$

where fre $_{\max } /$ fre $_{\text {min }}$ are the maximum/minimum values of the bat signal frequency, $v_{i}^{k}$ is the velocity of the $i^{\text {th }}$ bat in $k^{\text {th }}$ iteration and $\rho$ is a random number in the range $[0,1]$. Each bat is also updated in another way. In this regard, for each bat, a random value $\left(\operatorname{rand}_{i}\right)$ is first produced. If $\operatorname{rand}_{i}$ is greater than the frequency rate of the relevant bat $r_{i}$ thus a new test solution is generated as follows:

$$
X_{\text {new }}=X_{\text {old }}+\varepsilon A^{k}
$$

where $\varepsilon$ is a random number in the range $[0,1]$. The above formulation is much similar to the particle swarm optimization (PSO) algorithm updating process. On the other hand, if rand $_{i}$ is less than $r_{i}$ then a new test solution is generated randomly. The generated test solution is accepted if both the bellow criteria are satisfied:

$$
\begin{aligned}
& {\left[\operatorname{rand}<A_{i}\right]} \\
& {\left[f\left(X_{i}\right)<f\left(X_{\text {gbest }}\right)\right]}
\end{aligned}
$$

In the above equation, rand is a random number in the range [0,1]. Also $X_{\text {gbest }}$ is the best bat found in the population. The process of updating $A_{i}^{k}$ and $r_{i}^{k}$ in each iteration is as follows:

$$
\begin{aligned}
A_{i}^{k+1} & =\alpha A_{i}^{k} \\
r_{i}^{k+1} & =r_{i}^{0}[1-\exp (-\gamma k)]
\end{aligned}
$$

where $\alpha$ and $\gamma$ are constant values of BA.

\subsection{Modified Bat Algorithm}

The original BA has good characteristics such as simple concept, easy implementation, fast convergence, generic application, etc. However, in order to empower the performance of the algorithm in face of complex multi-objective optimization problems, a new modification is proposed in this section. The main idea is to increase the diversity of the BA population. This process is simulated such that the worst bat should move toward the best global bat which is found with more acceleration than the bat with better position. In fact, the acceleration movement of each bat in the population toward $X_{\text {gbest }}$ is determined according to its distance from $X_{\text {gbest }}$. Therefore after updating the BA population as described in Section 5.1, the proposed modification is applied. In this regard, the in each iteration and for each bat $X_{i}$, the Cartesian distance of the $X_{i}$ from the best bat in the population $X_{\text {gbest }}$ is calculated.

$$
\begin{aligned}
& D_{i, \text { gbest }}=\left\|X_{\text {gbest }}-X_{i}\right\|=\sqrt{\sum_{k=1}^{d}\left(x_{\text {gbest }, k}-x_{i, k}\right)} \\
& X_{\text {gbest }}=\left[x_{\text {gbest }, 1}, x_{\text {gbest }, 2}, \cdots, x_{\text {gbest }, d}\right] \\
& X_{i}=\left[x_{i, 1}, x_{i, 2}, \cdots, x_{i, d}\right]
\end{aligned}
$$

where $d$ is the length of the control vector $X$. Now, an attractiveness parameter $\beta$ is defined for $X_{\text {gbest }}$ as follows:

$$
\beta=\beta_{0} \exp \left(-D_{i, \text { gbest }}\right)
$$

where $\beta$ is the attractiveness of a bat firefly in the distance zero. The above formulation is a monotonically 
decreasing function. As it can be seen, as much the distance of $X_{i}$ is from $X_{g \text { best }}$, the lower value the variable $\beta$ will take. This formulation is useful to reach a tradeoff in the updating process. Now, the position of the bat $X_{i}$ is updated as follows:

$$
\begin{aligned}
& X_{\text {new }}=\beta X_{i}+(1-\beta) X_{\text {gbest }}+u_{k} \\
& u_{k}=\pi\left(\operatorname{rand}(\cdot)-\frac{1}{2}\right)
\end{aligned}
$$

where $\pi$ is the absorption coefficient which is employed to control the brightness reduction rate (light intensity).

The first two terms of the above equation are used to balance between the $X_{i}$ and $X_{\text {gbest }}$ and the third term is used for random movement in the search space.

\subsection{Fuzzy Interactive}

As mentioned before, the problem which is investigated here is a kind of multi-objective optimization problem with several equality and inequality constraints. As the result of the nature of the multi-objective optimization problems, improving any of the objective functions may result in destroying the optimal value of the other objective function. Therefore, here a sufficient multi-objective framework based on interactive fuzzy satisfying method is proposed to handle both of the objective functions. Therefore, by the use of the fuzzy set theory, the below equation is employed:

$$
F(X)=\min _{x \in \Omega}\left\{\max _{i=1, \cdots, n}\left|\mu_{r e f, i}-\mu_{f, i}(X)\right|\right\}
$$

In the above equation, $\mu_{f, i}(X)$ is the fuzzy membership function (trapezoidal membership function) and $\mu_{r e f, i}$ is the satisfying degree of the $f_{i}(X)$. The variable $n$ shows the number of objective functions. By the use of the above formulation, the operator can apply his/her preferences and experiences to satisfy each objective function individually.

\section{Application}

In order to apply the proposed stochastic method on the DSTATCOM optimal allocation and sizing, the following steps should be implemented:

Step 1: Define the input data including the network data, the DSTATCOM data, the algorithm data, etc.

Step 2: Convert the constrained Multi-objective opti- mization problem to its equivalent unconstraint one using the idea of penalty factors as follows (see Equation (20) below):

where $h_{j}(X)$ is the $j^{\text {th }}$ equality constraint and $g_{j}(X)$ is the $j^{\text {th }}$ inequality constraint. Also, $N_{e q}$ and $N_{\text {ueq }}$ show the number of equality and inequality constraints respectively. The penalty factors $L_{1}$ and $L_{2}$ are employed to meet the equality and inequality constraints. In this paper, the values of penalty factors are assumed $10^{8}$.

Step 3: Generate the initial bat population randomly. Each bat indicates a promising optimal location and size for the DSTATCOM devices in the network.

Step 4: Calculate the fitness function. In this step, the stochastic load flow based on $2 \mathrm{~m}$ PEM is run to calculate the expected value of the active power losses and the voltage deviation objective functions for each solution or bat.

Step 5: Apply the interactive fuzzy satisfying method to convert the multi-objective optimization problem to the equivalent single objective one. Here the membership function value of each objective function is calculated.

Step 6: Choose the best bat in the population as $X_{\text {gbest }}$

Step 7: Update the bat population as described in Section 5.1 .

Step 8: Apply the proposed modification as described in Section 5.2. Here again the entire population is updated.

Step 9: Check the termination criterion. The termination criterion can be the maximum number of iterations to update the BA population or a specific value which the objective function should reach to. If it is satisfied then finish the algorithm otherwise return to step 6 .

\section{Simulation Results}

In this section the simulation results of applying the proposed method on the test system is shown. The test system is the IEEE 69-bus radial distribution system which is chosen from a part of PG\&E distribution system. The nominal voltage of the system is $12.66 \mathrm{kV}$. The amounts of total active and reactive loads which are supplied by the system are $3802.19 \mathrm{~kW}$ and $2694.59 \mathrm{kVar}$ respectively. The single line diagram of the test system is shown in Figure 3. The complete data of the test system can be found in [17].

The initial size of the BA population is chosen 15 individuals. The termination criterion is supposed 100 iterations. The reason for this decision is that there is no

$$
U(X)=\left[\begin{array}{l}
f_{1}(X)+L_{1} \sum_{j=1}^{N_{\text {eq }}}\left(h_{j}(X)\right)^{2}+L_{2}\left(\sum_{j=1}^{\text {Nueq }}\left(\operatorname{Max}\left[0,-g_{j}(X)\right]\right)^{2}\right) \\
f_{2}(X)+L_{1} \sum_{j=1}^{N_{e q}}\left(h_{j}(X)\right)^{2}+L_{2}\left(\sum_{j=1}^{\text {Nueq }}\left(\operatorname{Max}\left[0,-g_{j}(X)\right]\right)^{2}\right)
\end{array}\right]
$$




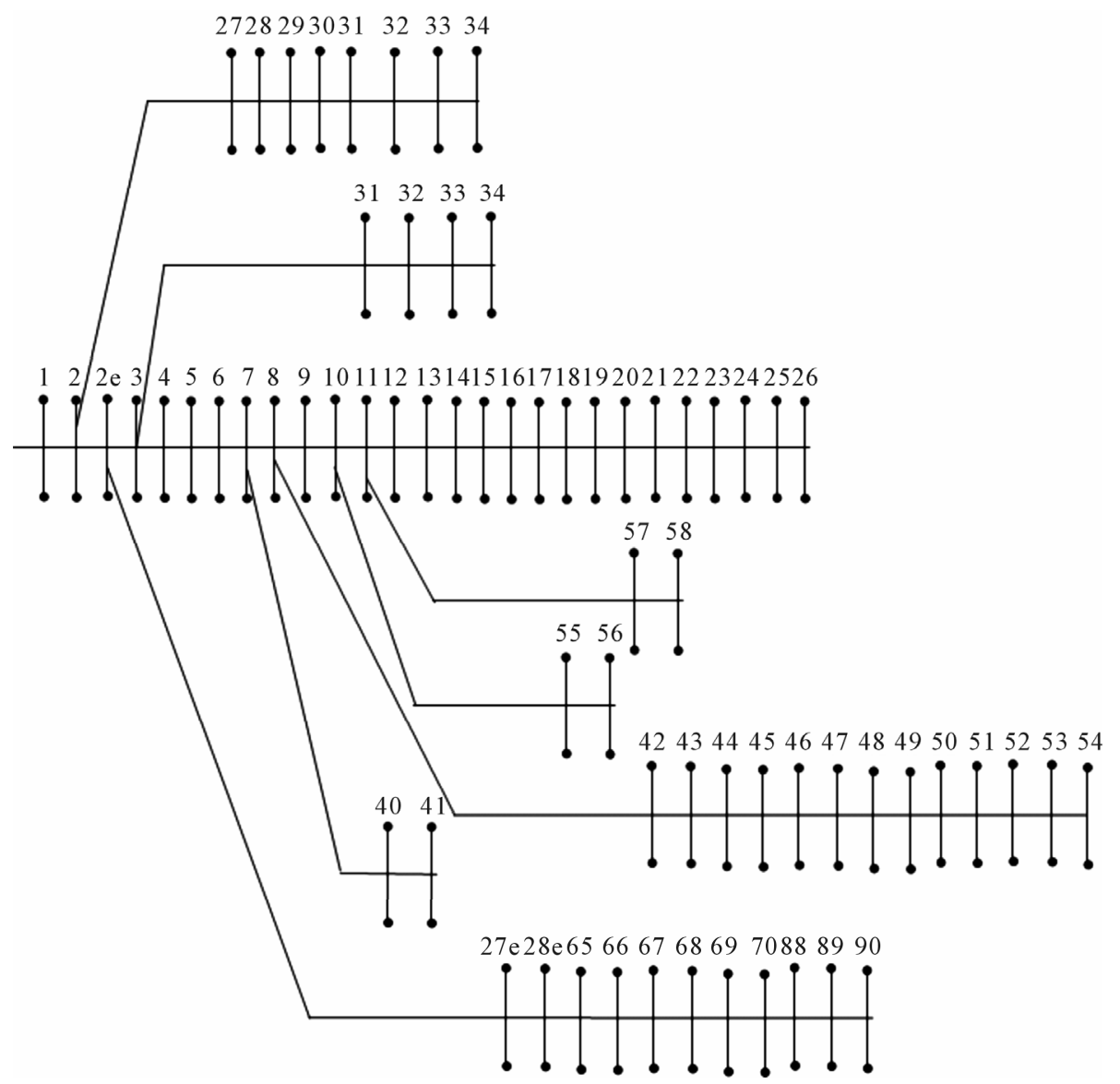

Figure 3. Single line diagram of the test system.

improvement in the objective function values after about 100 iterations. For better comparison, the analysis is implemented in both the deterministic and stochastic frameworks. Also, there are four different cases defined which are shown in the table:

Case 1: Initial system condition neglecting DSTATCOM

Case 2: DSTATCOM allocation and sizing for optimizing the total active power losses objective function

Case 3: DSTATCOM allocation and sizing for optimizing the maximum voltage deviation objective function

Case 4: DSTATCOM allocation and sizing for optimizing both of the total active power losses and voltage deviation by the proposed interactive fuzzy satisfying technique

In the first step, in order to see the satisfying performance of the proposed modified BA, a complete comparison is made with the other well-known methods in the area. The simulation results are shown in Table 1.

According to the Table 1, the superiority of the proposed method over the other well-known methods is evident. In order to see the positive effect of considering DSTATCOM in the system clearly, two different operating conditions (OCs) are defined. In the first OC, just 1
DSTATCOM is allocated in the system while in the second OC two DSTATCOMs are allocated. The maximum capacity of each DSTATCOM is $3 \mathrm{MW}$. The simulation results for the OC 1 and for all the four Cases in the deterministic framework (neglecting the uncertainty effects) are shown in Table 2. The word NG in the table means that negative power loss reduction (increase in the power loss) has happened.

As it can be seen from Table 2, in the Case 2, the amount of active power losses is reduced effectively. This amount of power loss reduction equals about 33.245 percent of the initial power losses which means notable value.

Also as it is seen, in this case, the voltage profile of the buses is improved as an indirect result of reducing resistive losses in the network. In the Case 3, the main target is optimizing the voltage deviation of the buses which has resulted to increasing the minimum voltage of the network. Here the amount of active power loss reduction is reduced to about 19.6460 percent. Finally, in the third case, the proposed interactive fuzzy satisfying method could reach proper tradeoff between the active power losses and the voltage deviation. In fact, as it is seen from Table 2, the proposed multi-objective formulation has 
Table 1. Simulation results of optimizing active power losses and voltage deviation objective function for 20 trails (OC 1).

\begin{tabular}{cccc}
\hline Items & Optimizing power losses & Optimizing Voltage Deviation & Mean CPU Time \\
\hline GA & 151.67002 & 0.04555011 & 11.358 \\
PSO & 150.23882 & 0.04533289 & 10.102 \\
Original BA & 150.23882 & 0.04500756 & 10.093 \\
Proposed MBA & 150.19693 & 0.04485347 & 8.876 \\
\hline
\end{tabular}

Table 2. Results obtained by optimal DSTATCOM allocation and sizing in the deterministic framework (OC 1).

\begin{tabular}{ccccc}
\hline Items & Case 1 & Case2 & Case 3 & Case 4 \\
\hline Active power losses $(\mathrm{kW})$ & 225.0 & 150.196936 & 269.204006 & 172.684083 \\
Loss reduction (\%) & $\ldots \ldots \ldots .$. & 33.245805 & NG & 23.2515185 \\
$\mathrm{~V}_{\min }(\mathrm{pu})$ & 0.90944095 & 0.93092967 & 0.95514652 & 0.941715813 \\
$\mathrm{~V}_{\max }(\mathrm{pu})$ & 1 & 1 & 1 & 1 \\
Voltage Deviation $(\mathrm{pu})$ & & 0.06907032 & 0.04485347 & 0.05828418 \\
DSTATCOM size $(\mathrm{MW})$ & $\ldots \ldots \ldots$ & 1.326 & 3 & 2.046 \\
DSTATCOM location & $\ldots \ldots \ldots .$. & 61 & 63 & 62 \\
\hline
\end{tabular}

optimized both of the objective functions suitably. Therefore, when the maximum bus voltage deviation of the system is improved to the appropriate value of $0.0582841 \mathrm{pu}$, the amount of power losses is reduced to the optimal value of $172.684 \mathrm{~kW}$. By comparing the optimal size of the DSTATCOM in different cases, it is deduced that better voltage profile is achieved for higher values of reactive power compensation by the DSTATCOM but with the cost of incremental losses. This result shows the necessity of accurate sizing and allocating of the DSTATCOM in the system.

Now, the stochastic analysis is utilized. In this analysis, the normal distribution system is supposed for all the active and reactive loads to model their uncertainty. The standard deviation of the probability density functions is assumed to be 0.05 . Table 3 shows the results of multiobjective optimization in both the deterministic and stochastic frameworks.

As it can be seen, considering the uncertainty in the analysis has resulted to incremental values in the objective functions. However, the standard deviation values of the objective functions in the stochastic framework are reduced. The less standard deviation values show more dependable optimal values near the real operating points.

Figure 4 shows the voltage profile enhancement before and after using 1 DSTATCOM in the system (case of multi-objective optimization).

According to the Figure 4, the role of using 1 DSTAT$\mathrm{COM}$ to enhance the power quality of the system is evident.

Now it is time to run the second scenario called OC 2.

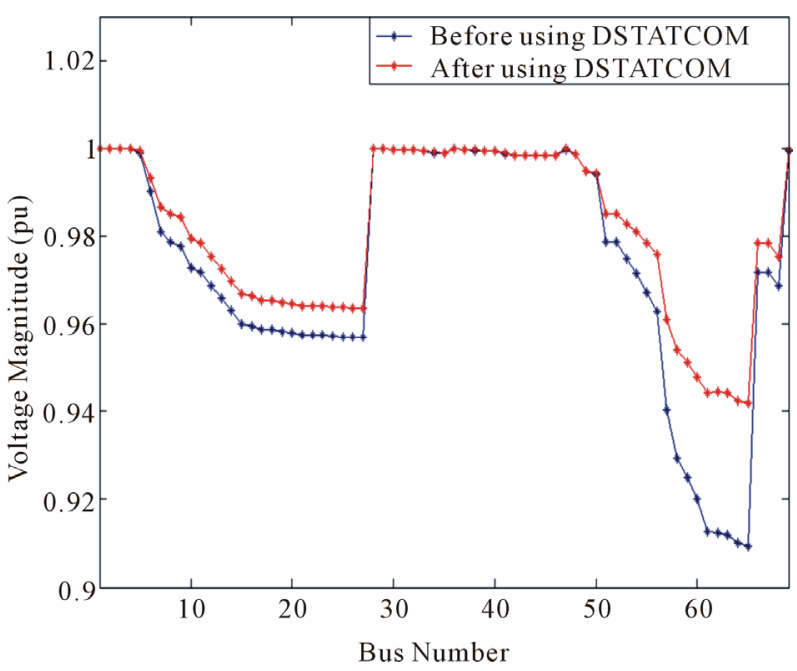

Figure 4. The voltage profile before and after using DSTATCOM in OC 1 (CASE 4 in the stochastic framework).

In this scenario, 2 DSTATCOM are allowed to be used in the system. The main target is to see the effect of using 1 more DSTATCOM on the optimal operating point of the network. The simulation results are shown in Table 4.

As it can be seen from Table 4, the existence of DSTATCOM in the system has improved the all the objective functions suitably. Nevertheless, by comparing the results of Table 2 with Table 4, it is seen that the choice of using the second DSTATCOM could not improve the values of the objective functions notably. In fact, once using just 1 DSTATCOM in the first scenario (OC 1) could reduce the amount of both the power losses 
Table 3. Results obtained by optimal DSTATCOM allocation and sizing in the stochastic framework (OC 1).

\begin{tabular}{cccc}
\hline Items & Item & Power Losses & Voltage Deviation \\
Deterministic Framework & Objective Function & 172.684083 & 0.05828418 \\
& Standard Deviation & 4.54322 & 0.001266 \\
Stochastic Framework & Objective Function & 173.948302 & 0.06028894 \\
& Standard Deviation & 3.98729 \\
\hline
\end{tabular}

Table 4. Results obtained by Optimal DSTATCOM allocation and sizing in the deterministic framework (OC 2).

\begin{tabular}{|c|c|c|c|c|}
\hline Items & Case 1 & Case 2 & Case 3 & Case 4 \\
\hline Active Power Losses (kW) & 225.0 & 144.8505116 & 653.0246131 & 172.474278 \\
\hline Loss Reduction (\%) & $\ldots \ldots \ldots$ & 35.6219948 & NG & 23.3447649 \\
\hline $\mathrm{V}_{\min }(\mathrm{pu})$ & 0.91309003 & 0.931319516 & 0.973002854 & 0.9421118440 \\
\hline $\mathrm{V}_{\max }(\mathrm{pu})$ & 1 & 1 & 1.000239326 & 1 \\
\hline Voltage Deviation (pu) & 0.08690929 & 0.068680483 & 0.026997145 & 0.05788815 \\
\hline DSTATCOM Size (MW) & a........ & $\begin{array}{l}17 \\
61\end{array}$ & $\begin{array}{l}57 \\
61\end{array}$ & $\begin{array}{l}54 \\
64\end{array}$ \\
\hline DSTATCOM Location & .......... & $\begin{array}{l}0.353 \\
1.273\end{array}$ & $\begin{array}{l}3 \\
3\end{array}$ & $\begin{array}{l}1.423 \\
1.477\end{array}$ \\
\hline
\end{tabular}

Table 5. Results obtained by optimal DSTATCOM allocation and sizing in the stochastic framework (OC 2).

\begin{tabular}{cccc}
\hline Items & Item & Power Losses & Voltage Deviation \\
\hline \multirow{2}{*}{ Deterministic Framework } & Objective Function & 144.8505116 & 0.0269971 \\
& Standard Deviation & 4.00128 & 0.000539 \\
& Objective Function & 173.948302 & 0.06028894 \\
Stochastic Framework & Standard Deviation & 3.78351 & 0.000421 \\
\hline
\end{tabular}

and voltage deviation effectively, the second DSTATCOM has just negligible effect to further improvement of these objective functions. Therefore, according to the expensive value of each DSTATCOM in the system, this amount of improvement in the objective functions is not economical and using the second DSTATCOM is not sensible. The simulation results of the stochastic framework are shown in Table 5.

As it is seen here again, the proposed stochastic method has improved the dependability of the final solutions by reducing the standard deviation values. Finally in Figure 5, the voltage profile before and after using the OC 2 is shown comparatively.

\section{Conclusion}

This paper investigated the optimal placement and sizing of the DSTATCOM device in the distribution systems. In this regard, a new stochastic optimization framework

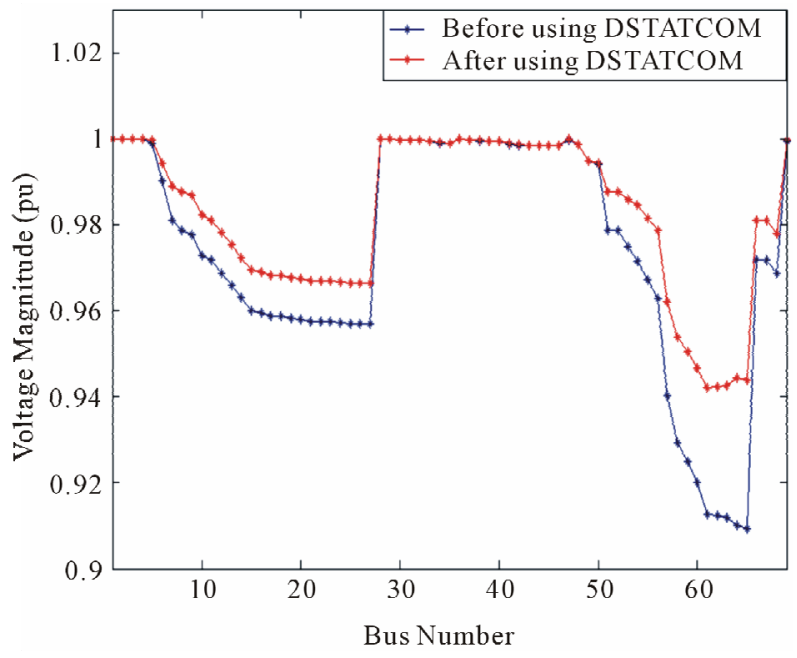

Figure 5. The voltage profile before and after using 2 DSTATCOMs in OC 2 (CASE 4 in the stochastic framework). 
based on the $2 \mathrm{~m}$ PEM and Modified BA was also proposed to solve the problem. The two significant objective functions including active power losses and the voltage deviation are handled using the idea of interactive fuzzy satisfying method. The simulation results showed that using the DSTATCOM as a well-known Facts device can improve the system condition effectively. Nevertheless, there is no direct relationship which states that increasing the number of DSTATCOM in the system can improve the objective functions. In fact, as it was seen from the simulation results, in the 69-bus IEEE test system, the amount of growth in the objective functions values for just 1 DSTATCOM is more than that of using 2 DSTATCOM. From the uncertainty point of view, the proposed stochastic framework could increase the dependability of the optimal solutions by reducing the standard deviation values.

\section{REFERENCES}

[1] A. Kavousi-Fard and M. R. Akbari-Zadeh, "Reliability Enhancement Using Optimal Feeder Reconfiguration," Neurocomputing, Vol. 106, No. 1, 2013, pp. 1-11. doi:10.1016/j.neucom.2012.08.033

[2] A. Kavousifard and H. Samet, "Consideration Effect of Uncertainty in Power System Reliability Indices Using Radial Basis Function Network and Fuzzy Logic Theory," Neurocomputing, Vol. 74, No. 17, 2011, pp. 3420- 3427. doi:10.1016/j.neucom.2011.05.017

[3] C. Sumpavakup and T. Kulworawanichpong, "Distribution Voltage Regulation under Three-Phase Fault by Using D-STATCOM," World Academy of Science, Engineering and Technology, Vol. 47, No. 1, 2008, pp. 238242.

[4] Z. Yang, C Shen, M. L. Crow and L. Zhang, "An Improved STATCOM Model for Power Flow Analysis," Power Engineering Society Summer Meeting, Vol. 2, Seattle, 16-20 July 2000, pp. 1121-1126.

[5] G. Ledwich and A. A. Ghosh, "Flexible DSTATCOM Operating in Voltage or Current Control Mode," IEE Proceedings-Generation Transmission and Distribution, Vol. 149, No. 2, 2002, pp. 215-224.

[6] I. Wasiak, R. Mienski, R. Pawelek and P. Gburczyk, "Application of DSTATCOM Compensators for Mitigation of Power Quality Disturbances in Low Voltage Grid with Distributed Generation," 9th International Conference on Electrical Power Quality and Utilizations, Barcelona, 911 October 2007, pp. 1-6.

[7] T. Niknam, A. Kavousifard, S. Tabatabaei and J. Aghae, "Optimal Operation Management of Fuel Cell/Wind/Pho- tovoltaic Power Sources Connected to Distribution Networks," Journal of Power Sources, Vol. 196, No. 20, 2011, pp. 8881-8896. doi:10.1016/i.jpowsour.2011.05.081

[8] L. L. Lai and J. T. Ma, “Application of Evolutionary Programming to Reactive Power Planning-Comparison with Nonlinear Programming Approach," IEEE Transactions on Power Systems, Vol. 12, No. 1, 1997, pp. 198-206. doi: $10.1109 / 59.574940$

[9] T. Niknam and A. Kavousifard, "Impact of Thermal Recovery and Hydrogen Production of Fuel Cell Power Plants on Distribution Feeder Reconfiguration," IET Generation Transmission \& Distribution, Vol. 6, No. 9, 2012, pp. 831-843. doi:10.1049/iet-gtd.2011.0775

[10] Z. Xu, Z. Y. Dong and K. P. Wong, "Genetic AlgorithmBased Spinning Reserve Dispatching in a Competitive Electricity Market," International Journal of Systems Science, Vol. 35, No. 13-14, 2004, pp. 847-853. doi: $10.1080 / 00207720412331303688$

[11] T. Niknam, A. Kavousifard and J. Aghaei, "ScenarioBased Multiobjective Distribution Feeder Reconfiguration Considering Wind Power Using Adaptive Modified Particle Swarm Optimization," IET Generation Transmission \& Distribution, Vol. 6, No. 4, 2012, pp. 236-247. doi:10.1049/iet-rpg.2011.0256

[12] D. Debaprya, "A Fuzzy Multi-Objective Approach for Network Reconfiguration of Distribution Systems," IEEE Transactions on Power Delivery, Vol. 21, No. 1, 2006, pp. 202-209. doi:10.1109/TPWRD.2005.852335

[13] T. Niknam, A. K. Fard and A. Seifi, "Distribution Feeder Reconfiguration Considering Fuel Cell/Wind/Photovoltaic power Plants," Journal of Renewable and Sustainable Energy, Vol. 37, No. 1, 2012, pp. 213-225. doi:10.1016/i.renene.2011.06.017

[14] S. Jazebi, S. H. Hosseinian and B. Vahidi, "DSTATCOM Allocation in Distribution Networks Considering Reconfiguration Using Differential Evolution Algorithm," Energy Conversion and Management, Vol. 52, No. 7, 2011, pp. 2777-2783. doi:10.1016/j.enconman.2011.01.006

[15] T. Niknam, A. K. Fard and A. Baziar, "Multi-Objective Stochastic Distribution Feeder Reconfiguration Problem Considering Hydrogen and Thermal Energy Production by Fuel Cell Power Plants," Energy, Vol. 4, No. 1, 2012, pp. 563-573. doi:10.1016/j.energy.2012.02.023

[16] A. Baziar, A. Kavoosi-Fard and J. Zare, "A Novel Self Adaptive Modification Approach Based on Bat Algorithm for Optimal Management of Renewable MG," Journal of Intelligent Learning Systems and Applications, Vol. 5, 2013, pp. 11-18. doi:10.4236/jilsa.2013.51002

[17] M. E. Baran and F. F. Wu, "optimal Capacitor Placement on Radial Distribution Systems," IEEE Transactions on Power Delivery, Vol. 4, No. 1, 1989, pp. 725-734. doi:10.1109/61.19265 